\title{
Existence and attractivity results for $\psi$-Hilfer hybrid fractional differential equations
}

\author{
FATIMA Si BACHIR ${ }^{1}$ \\ SAÏD ABBAS ${ }^{2}$ \\ MAAMAR BENBACHIR ${ }^{3}$ \\ MOUFFAK BENCHOHRA 4 \\ Gaston M. N'GuÉRÉKATA ${ }^{5}$ \\ 1 Laboratory of Mathematics and Applied \\ Sciences, University of Ghardaia, 47000, \\ Algeria. \\ sibachir.fatima@univ-ghardaia.dz.com \\ 2 Department of Mathematics, University \\ of Saïda-Dr. Moulay Tahar, P.O. Box \\ 138, EN-Nasr, 20000 Saïda, Algeria. \\ said.abbas@univ-saida.dz \\ 3 Department of Mathematics, Saad \\ Dahlab Blida1, University of Blida, \\ Algeria. \\ mbenbachir2001@gmail.com \\ ${ }^{4}$ Laboratory of Mathematics, Djillali \\ Liabes University of Sidi Bel-Abbès, P.O. \\ Box 89, Sidi Bel-Abbès 22000, Algeria. \\ benchohra@yahoo.com \\ 5 NEERLab, Department of \\ Mathematics, Morgan State University, \\ 1700 E. Cold Spring Lane, Baltimore \\ M.D. 21252, USA. \\ gaston.nguerekata@morgan.edu
}

\section{ABSTRACT}

In this work, we present some results on the existence of attractive solutions of fractional differential equations of the $\psi$-Hilfer hybrid type. The results on the existence of solutions are a consequence of the Schauder fixed point theorem. Next, we prove that all solutions are uniformly locally attractive.

\section{RESUMEN}

En este trabajo, presentamos algunos resultados sobre la existencia de soluciones atractivas de ecuaciones diferenciales fraccionarias de tipo $\psi$-Hilfer híbridas. Los resultados de existencia de soluciones son consecuencia del teorema de punto fijo de Schauder. A continuación, probamos que todas las soluciones son uniformemente localmente atractivas.

Keywords and Phrases: $\psi$-Hilfer fractional derivative; Schauder fixed-point Theorem; uniformly locally attractive.

2020 AMS Mathematics Subject Classification: 26A33, 34A08.

\section{(cc) BY-NC}

Accepted: 01 March, 2021

Received: 12 November, 2020
(C)2021 F. Si bachir et al. This open access article is licensed under a Creative Commons Attribution-NonCommercial 4.0 International License. 


\section{Introduction}

The theory of derivatives and integrals to a real or complex order is none other than the fractional theory which began in 1695 between G.A. de L'Hospital and G.W. Leibniz. The fractional integration and differentiation go back to Leibniz, Riemann, Liouville, Abel, Weyl, and Riesz [27]. Many monographs to which the reader can refer such as Abbas et al. [1, 5, 6], Diethelm [13], Kilbas et al. [17], Oldham et al. [22], Podlubny [23], Samko et al. [24], Zhou [32, 33], Zhou et al. [34] and the works by Abbas and Benchohra [2], Lakshmikantham et al. [19, 20, 21]. Recently several works have been done concerning hybrid fractional differential equations see [9, 12, 14, 26, 31], and the references therein.

Functional $\psi$ - fractional differential equations received a great importance in applied mathematics and other sciences, see $[8,16,18,25,28,29,30]$, and the references therein.

Some interesting results on existence and attractivity have been obtained in $[3,4,7]$. In this work, we are interested in the existence and attractivity of solutions for the following problem

$$
\left\{\begin{array}{lr}
D_{0^{+}}^{\lambda, \sigma \psi} \frac{u(t)}{v(t, u(t))}=w(t, u(t)) ; \text { a.e. } & t \in \mathbb{R}_{+}, \\
\left.(\psi(t)-\psi(0))^{1-\varsigma} u(t)\right|_{t=0}=u_{0} ; & u_{0} \in \mathbb{R},
\end{array}\right.
$$

where $\mathbb{R}_{+}:=[0,+\infty), 0<\lambda<1,0 \leq \sigma \leq 1, \varsigma=\lambda+\sigma(1-\lambda),{ }^{H} D_{0^{+}}^{\lambda, \sigma ; \psi}$ is the $\psi$-Hilfer fractional derivative of order $\lambda$ and type $\sigma, v: \mathbb{R}_{+} \times \mathbb{R} \rightarrow \mathbb{R}^{*}$ and $w: \mathbb{R}_{+} \times \mathbb{R} \rightarrow \mathbb{R}$, are given functions.

\section{Special cases:}

- For $\sigma=0, \psi(t)=t, u_{0}=0$, we will get nonlinear hybrid FDEs of the form

$$
\left\{\begin{array}{l}
{ }^{R L} D_{0^{+}}^{\lambda}\left[\frac{u(t)}{v(t, u(t))}\right]=w(t, u(t)), \text { a.e. } t \in \mathbb{R}_{+}, \\
u(0)=0 .
\end{array}\right.
$$

- For $\lambda=1, \sigma=1, \psi(t)=t$, we will get nonlinear integer order hybrid differential equations of the form

$$
\left\{\begin{array}{l}
\frac{d}{d t}\left[\frac{u(t)}{v(t, u(t))}\right]=w(t, y(t)), \text { a.e. } t \in \mathbb{R}_{+}, \\
u(0)=u_{0} \in \mathbb{R} .
\end{array}\right.
$$

For $v=1$, we will get nonlinear $\psi$-Hilfer FDEs of the form

$$
\left\{\begin{array}{l}
{ }^{H} D_{0^{+}}^{\lambda, \sigma ; \psi} u(t)=w(t, y(t)), \text { a.e. } t \in \mathbb{R}_{+}, \\
\left.(\psi(t)-\psi(0))^{1-\varsigma} u(t)\right|_{t=0}=u_{0} \in \mathbb{R} .
\end{array}\right.
$$

- For $v=1, \sigma=0$ (in this case $\varsigma=\lambda$ ), $\psi(t)=t$, we will get nonlinear FDEs involving RiemannLiouville fractional derivative

$$
{ }^{R L} D_{0^{+}}^{\lambda} u(t)=w(t, y(t)) \text {, a.e. } t \in \mathbb{R}_{+} .
$$




\section{Preliminaries}

Let $\psi:[a, b] \rightarrow \mathbb{R}$ be an increasing differentiable function such that $\psi^{\prime}(t) \neq 0$, for all $t \in[a, b]$, $(-\infty \leq a<b \leq+\infty)$. Define on $[a, b],(0<a<b<\infty)$ the weighted space

$$
C_{\varsigma \psi[a, b]}=\left\{\tau:(a, b] \rightarrow \mathbb{R}:(\psi(t)-\psi(a))^{\varsigma} \tau(t) \in C[a, b]\right\}, \quad 0 \leq \varsigma<1,
$$

with the norm

$$
\|\tau\|_{C_{\varsigma ;}[a, b]}=\left\|(\psi(t)-\psi(a))^{\varsigma} \tau(t)\right\|_{C[a, b]}=\max \left\{\left|(\psi(t)-\psi(a))^{\varsigma} \tau(t)\right|: t \in[a, b]\right\},
$$

where $C([a, b])$ denotes the Banach space of all real continuous functions on $[a, b]$.

Let $B C:=B C\left(\mathbb{R}_{+}\right)$be the Banach space of all bounded and continuous functions from $\mathbb{R}_{+}$ into $\mathbb{R}$. By $B C_{\varsigma}:=B C_{\varsigma}\left(\mathbb{R}_{+}\right)$, we denote the weighted space of all bounded and continuous functions defined by $B C_{\varsigma}=\left\{\phi: \mathbb{R}_{+} \rightarrow \mathbb{R}:(\psi(t)-\psi(0))^{1-\varsigma} \phi(t) \in B C\right\}$, with the norm

$$
\|\phi\|_{B C_{\varsigma}}:=\sup _{t \in \mathbb{R}_{+}}\left|(\psi(t)-\psi(0))^{1-\varsigma} \phi(t)\right| .
$$

Let us recall some definitions and properties of fractional calculus.

Definition 2.1. [17] The left-sided $\psi$-Riemann-Liouville fractional integral and fractional derivative of order $\lambda,(n-1<\lambda<n)$ for an integrable function $\phi:[a, b] \rightarrow \mathbb{R}$ with respect to another function $\psi:[a, b] \rightarrow \mathbb{R}$, that is an increasing differentiable function such that $\psi^{\prime}(t) \neq 0$, for all $t \in[a, b],(-\infty \leq a<b \leq+\infty)$, are respectively defined as follows:

$$
I_{a^{+}}^{\lambda ; \psi} \phi(t)=\frac{1}{\Gamma(\lambda)} \int_{a}^{t} \psi^{\prime}(s)(\psi(t)-\psi(s))^{\lambda-1} \phi(s) d s,
$$

and

$$
D_{a+}^{\lambda ; \psi} \phi(t)=\left(\frac{1}{\psi^{\prime}(t)} \frac{d}{d t}\right)^{n} I_{a^{+}}^{n-\alpha ; \psi} \phi(t)=\frac{1}{\Gamma(n-\lambda)}\left(\frac{1}{\psi^{\prime}(t)} \frac{d}{d t}\right)^{n} \int_{a}^{t} \psi^{\prime}(s)(\psi(t)-\psi(s))^{n-\lambda-1} \phi(s) d s,
$$

where $\Gamma(\cdot)$ is the Euler gamma function defined by

$$
\Gamma(\delta)=\int_{0}^{\infty} e^{-t} t^{\delta-1} d t, \quad \delta>0 .
$$

Definition 2.2. [10] The left-sided $\psi$-Caputo fractional derivative of function $\chi \in C^{n}[a, b]$, $(n-1<\lambda<n) n=[\alpha]+1$ with respect to another function $\psi$ is defined by

$$
{ }^{c} D_{a^{+}}^{\lambda ; \psi} \phi(t)=I_{a^{+}}^{n-\lambda ; \psi}\left(\frac{1}{\psi^{\prime}(t)} \frac{d}{d t}\right)^{n} \phi(t)=\frac{1}{\Gamma(n-\lambda)} \int_{a}^{t} \psi^{\prime}(s)(\psi(t)-\psi(s))^{n-\lambda-1} \phi_{\psi}^{[n]}(s) d s,
$$

where $\phi_{\psi}^{[n]}(t)=\left(\frac{1}{\psi^{\prime}(t)} \frac{d}{d t}\right)^{n} \phi(t)$ and $\psi$ defined as in Definition $\mathbb{Q}$. Moreover, the $\psi-$ Caputo fractional derivative of function $\phi \in A C^{n}[a, b]$ is determined as

$$
{ }^{c} D_{a^{+}}^{\lambda ; \psi} \phi(t)=D_{a^{+}}^{\lambda ; \psi}\left[\phi(t)-\sum_{k=0}^{n-1} \frac{\left[\frac{1}{\psi^{\prime}(t)} \frac{d}{d t}\right]^{k} \phi(a)}{k !}(\psi(t)-\psi(a))^{k}\right] .
$$


Definition 2.3. [29] Let $n-1<\lambda<n, n \in \mathbb{N}$, with $[a, b],-\infty \leq a<b \leq+\infty$, and $\psi \in C^{n}([a, b], \mathbb{R})$ a function such that $\psi(t)$ is increasing and $\psi^{\prime}(t) \neq 0$, for all $t \in[a, b]$. The $\psi$-Hilfer fractional derivative (left-sided) of function $\phi \in C^{n}([a, b], \mathbb{R})$ of order $\lambda$ and type $\sigma \in[0,1]$ is determined as

$$
D_{a^{+}}^{\lambda, \sigma ; \psi} \phi(t)=I_{a^{+}}^{\sigma(n-\lambda) ; \psi}\left[\frac{1}{\psi^{\prime}(t)} \frac{d}{d t}\right]^{n} I_{a^{+}}^{(1-\sigma)(n-\lambda) ; \psi} \phi(t), t>a .
$$

In other way

$$
D_{a^{+}}^{\lambda, \sigma ; \psi} \phi(t)=I_{a^{+}}^{\sigma(n-\lambda) ; \psi} D_{a^{+}}^{\gamma ; \psi} \phi(t), t>a
$$

where

$$
D_{a^{+}}^{\gamma ; \psi} \phi(t)=\left[\frac{1}{\psi^{\prime}(t)} \frac{d}{d t}\right]^{n} I_{a^{+}}^{(1-\sigma)(n-\lambda) ; \psi} \phi(t) .
$$

In particular, the $\psi$-Hilfer fractional derivative of order $\lambda \in(0,1)$ and type $\sigma \in[0,1]$, can be written in the following form

$$
\begin{aligned}
D_{a^{+}}^{\lambda, \sigma \psi} \phi(t) & =\frac{1}{\Gamma(\varsigma-\lambda)} \int_{a}^{t}(\psi(t)-\psi(s))^{\varsigma-\lambda-1} D_{a^{+}}^{\gamma ; \psi} \phi(s) d s \\
& =I_{a^{+}}^{\varsigma-\lambda ; \psi} D_{a^{+}}^{\varsigma ; \psi} \phi(t),
\end{aligned}
$$

where $\varsigma=\lambda+\sigma-\lambda \sigma$, and $D_{a^{+}}^{\varsigma ; \psi} \phi(t)=\left[\frac{1}{\psi^{\prime}(t)} \frac{d}{d t}\right] I_{a^{+}}^{1-\varsigma ; \psi} \phi(t)$.

Lemma 2.4. [29] Let $\lambda>0,0 \leq \varsigma<1$ and $\phi \in L^{1}(a, b)$. Then

$$
I_{a^{+}}^{\lambda ; \psi} I_{a^{+}}^{\sigma ; \psi} \phi(t)=I_{a^{+}}^{\lambda+\sigma ; \psi} \phi(t), \text { a.e. } t \in[a, b] .
$$

In particular (i) if $\phi \in C_{\varsigma ; \psi}[a, b]$, then $I_{a+}^{\lambda ; \psi} I_{a+}^{\sigma ; \psi} \phi(t)=I_{a+}^{\lambda+\sigma ; \psi} \phi(t), t \in(a, b]$.

(ii) If $\phi \in C[a, b]$, then $I_{a^{+}}^{\lambda ; \psi} I_{a^{+}}^{\sigma ; \psi} \phi(t)=I_{a^{+}}^{\lambda+\sigma ; \psi} \phi(t), t \in[a, b]$.

Lemma 2.5. [29] Let $\lambda>0,0 \leq \sigma \leq 1$ and $0 \leq \varsigma<1$. If $h \in C_{\varsigma ; \psi}[a, b]$ then

$$
D_{a^{+}}^{\lambda, \sigma ; \psi} I_{a^{+}}^{\lambda ; \psi} \phi(t)=\phi(t), t \in(a, b] .
$$

If $\phi \in C^{1}[a, b]$ then

$$
D_{a+}^{\lambda, \sigma ; \psi} I_{a+}^{\alpha ; \psi} \phi(t)=\phi(t), t \in[a, b] .
$$

Lemma 2.6. Let $\lambda>0,0 \leq \varsigma<1$ and $\phi \in C_{\varsigma ; \psi}[a, b]$. If $\lambda>\varsigma$, then $I_{a^{+}}^{\lambda ; \psi} \phi \in C[a, b]$ and

$$
I_{a^{+}}^{\lambda ; \psi} \phi(a)=\lim _{t \rightarrow a^{+}} I_{a^{+}}^{\lambda ; \psi} \phi(t)=0 .
$$

Lemma 2.7. [29] Let $\phi \in C^{n}[a, b], n-1<\lambda<n, 0 \leq \sigma \leq 1$, and $\varsigma=\lambda+\sigma-\lambda \sigma$. Then for all $t \in(a, b]$

$$
I_{a^{+}}^{\lambda ; \psi} D_{a^{+}}^{\lambda, \sigma ; \psi} \phi(t)=\phi(t)-\sum_{k=1}^{n} \frac{[\psi(t)-\psi(a)]^{\varsigma-k}}{\Gamma(\varsigma-k+1)} \phi_{\psi}^{(n-k)} I_{a^{+}}^{(1-\sigma)(n-\lambda) ; \psi} \phi(a) .
$$

In particular, if $0<\lambda<1$, we have

$$
I_{a^{+}}^{\lambda ; \psi} D_{a^{+}}^{\lambda, \sigma ; \psi} \phi(t)=\phi(t)-\frac{[\psi(t)-\psi(a)]^{\varsigma-1}}{\Gamma(\varsigma)} I_{a^{+}}^{(1-\sigma)(1-\lambda) ; \psi} \phi(a) .
$$


Moreover, if $\phi \in C_{1-\varsigma ; \psi}[a, b]$ and $I_{a^{+}}^{1-\varsigma ; \psi} \phi \in C_{1-\varsigma ; \psi}^{1}[a, b]$ such that $0<\varsigma<1$. Then for all $t \in(a, b]$

$$
I_{a^{+}}^{\varsigma ; \psi} D_{a^{+}}^{\varsigma ; \psi} \phi(t)=\phi(t)-\frac{[\psi(t)-\psi(a)]^{\gamma-1}}{\Gamma(\varsigma)} I_{a^{+}}^{1-\varsigma ; \psi} \phi(a) .
$$

We deduce from the above lemma the following lemmas:

Lemma 2.8. [18] Let $v \in C\left(\Upsilon \times \mathbb{R}, \mathbb{R}^{*}\right) ; \Upsilon:=[0, d], d>0, \kappa \in C_{1-\zeta, \psi}(\Upsilon)$. Then the problem

$$
\left\{\begin{array}{l}
D_{0^{+}}^{\lambda, \sigma ; \psi} \frac{u(t)}{v(t, u(t))}=\kappa(t), \text { a.e. } \quad t \in \Upsilon . \\
\left.(\psi(t)-\psi(0))^{1-\varsigma} u(t)\right|_{t=0}=u_{0}, \quad u_{0} \in \mathbb{R}
\end{array}\right.
$$

has a unique solution given by

$$
u(t)=v(t, u(t))\left\{\frac{u_{0}}{v(0, u(0))}(\psi(t)-\psi(0))^{\varsigma-1}+I_{0^{+}}^{\lambda ; \psi} \kappa(t)\right\} .
$$

Lemma 2.9. Let $v \in C\left(\Upsilon \times \mathbb{R}, \mathbb{R}^{*}\right), w: \Upsilon \times \mathbb{R} \rightarrow \mathbb{R}$ be a function such that $w(\cdot, u(\cdot)) \in B C_{\varsigma}$ for any $u \in B C_{\varsigma}$. Then the problem (1.1) is equivalent to the problem of obtaining the solutions of the integral equation

$$
u(t)=v(t, u(t))\left\{\frac{u_{0}}{v(0, u(0))}(\psi(t)-\psi(0))^{\varsigma-1}+I_{0^{+}}^{\lambda ; \psi} w(\cdot, u(\cdot))(t)\right\} .
$$

Let $\varnothing \neq \Lambda \subset B C$ and let $K: \Lambda \rightarrow \Lambda$. We consider the solution of the equation

$$
(K u)(t)=u(t)
$$

We introduce the concept of attractivity of solutions for equation (2.1).

Definition 2.10. Solutions of equation (2.1) are locally attractive if there exists a ball $B\left(u_{0}, \mu\right)$ in the space $B C$ such that, for any solutions $\tau=\tau(t)$ and $\xi=\xi(t)$ of equations (2.1) that belong to $B\left(u_{0}, \mu\right) \cap \Lambda$, we can write

$$
\lim _{t \rightarrow \infty}(\tau(t)-\xi(t))=0
$$

If the limit (2.2) is uniform with respect to $B\left(u_{0}, \mu\right) \cap \Lambda$, then the solutions of equation (2.1) are said to be uniformly locally attractive (or, equivalently, that the solutions of (2.1) are locally asymptotically stable).

Lemma 2.11. [11] Let $M \subset B C$. Then $M$ is relatively compact in $B C$ if the following conditions are satisfied:

(a) $M$ is uniformly bounded in $B C$;

(b) the functions belonging to $M$ are almost equicontinuous in $\mathbb{R}_{+}$, i.e., equicontinuous on every compact set in $\mathbb{R}_{+}$; 
(c) the functions from $M$ are equiconvergent, i.e., given $\varepsilon>0$, there exists $L(\varepsilon)>0$ such that

$$
\left|u(t)-\lim _{t \rightarrow \infty} u(t)\right|<\varepsilon
$$

for any $t \geq L(\varepsilon)$ and $u \in M$.

Theorem 2.12. (Schauder Fixed-Point Theorem [15]). Let $F$ be a Banach space, let $U$ be a nonempty bounded convex and closed subset of $F$, and let $K: U \rightarrow U$ be a compact and continuous map. Then $K$ has at least one fixed point in $U$.

\section{$3 \quad$ Existence and Attractivity Results}

Definition 3.1. A measurable function $u \in B C_{\varsigma}$ is a solution of problem (1.1) if it verifies the initial condition $\left.(\psi(t)-\psi(0))^{1-\varsigma} u(t)\right|_{t=0}=u_{0}$ and the equation $D_{0^{+}}^{\lambda, \sigma ; \psi} \frac{u(t)}{v(t, u(t))}=w(t, u(t))$ on $\mathbb{R}_{+}$.

We will give the following hypotheses:

$\left(H_{1}\right)$ The function $t \mapsto w(t, u)$ is measurable on $\mathbb{R}_{+}$for each $u \in B C_{\varsigma}$, the function $u \mapsto w(t, u)$ is continuous on $B C_{\varsigma}$ for a.e. $t \in \mathbb{R}_{+}$, and the function $v$ is bounded such that $u \mapsto v(t, u)$ is continuous.

$\left(H_{2}\right)$ There exists a continuous function $T: \mathbb{R}_{+} \rightarrow \mathbb{R}_{+}$such that for a.e. $t \in \mathbb{R}_{+}$and each $u \in \mathbb{R}$,

$$
|w(t, u)| \leq \frac{T(t)}{1+|u|}
$$

and

$$
\lim _{t \rightarrow \infty}(\psi(t)-\psi(0))^{1-\varsigma}\left(I_{0^{+}}^{\lambda ; \psi} T\right)(t)=0 .
$$

Set

$$
T^{*}=\sup _{t \in \mathbb{R}_{+}}(\psi(t)-\psi(0))^{1-\varsigma}\left(I_{0^{+}}^{\lambda ; \psi} T\right)(t)<\infty
$$

Now we present a theorem on the existence and attractivity of solutions of the problem (1.1).

Theorem 3.2. Assume that the hypotheses $\left(H_{1}\right)$ and $\left(H_{2}\right)$ hold. Then the problem (1.1) has at least one solution defined on $\mathbb{R}_{+}$and the solutions of problem (1.1) are uniformly locally attractive.

Proof. Consider the operator $K$ such that, for any $u \in B C_{\varsigma}$,

$$
(K u)(t)=v(t, u(t))\left\{\frac{u_{0}}{v(0, u(0))}(\psi(t)-\psi(0))^{\varsigma-1}+\frac{1}{\Gamma(\lambda)} \int_{0}^{t} \psi^{\prime}(s)(\psi(t)-\psi(s))^{\lambda-1} w(s, u(s)) d s\right\} .
$$


Let $L$ be a bound of the function $v$. For any $u \in B C_{\varsigma}$, and for each $t \in \mathbb{R}_{+}$, we have

$$
\begin{aligned}
& \left|(\psi(t)-\psi(0))^{1-\varsigma}(K u)(t)\right| \\
\leq & |v(t, u(t))|\left\{\left|\frac{u_{0}}{v(0, u(0))}\right|+\frac{(\psi(t)-\psi(0))^{1-\varsigma}}{\Gamma(\lambda)} \int_{0}^{t} \psi^{\prime}(s)(\psi(t)-\psi(s))^{\lambda-1}|w(s, u(s))| d s\right\} \\
\leq & |v(t, u(t))|\left\{\left|\frac{u_{0}}{v(0, u(0))}\right|+\frac{(\psi(t)-\psi(0))^{1-\varsigma}}{\Gamma(\lambda)} \int_{0}^{t} \psi^{\prime}(s)(\psi(t)-\psi(s))^{\lambda-1} T(s) d s\right\} \\
\leq & L\left\{\left|\frac{u_{0}}{v(0, u(0))}\right|+T^{*}\right\} \\
:= & R_{*} .
\end{aligned}
$$

So

$$
\mid K(u) \|_{B C} \leq R_{*}
$$

Therefore, $K(u) \in B C_{\varsigma}$. Since, the map $K(u)$ is continuous on $\mathbb{R}_{+}$; for any $u \in B C_{\varsigma}$, and $K\left(B C_{\varsigma}\right) \subset$ $B C_{\varsigma}$, then the operator $K$ maps $B C_{\varsigma}$ into itself. Furthermore, equation (3.1) implies that the operator $K$ transforms the ball

$$
B_{R_{*}}:=B\left(0, R_{*}\right)=\left\{v \in B C_{\varsigma}:\|v\|_{B C_{\varsigma}} \leq R_{*}\right\}
$$

into itself. From Lemma 2.9 the solution of problem (1.1) is reduced to finding the solutions of the operator equation $K(u)=u$. We show that the operator $K: B C_{\varsigma} \rightarrow B C_{\varsigma}$ satisfies all assumptions of Theorem 2.12. The proof is divided into several steps:

Step 1. $K$ is continuous.

Let $\left\{u_{n}\right\}_{n \in N}$ be a sequence such that $u_{n} \rightarrow u$ in $B_{R_{*}}$.

Then, for each $t \in \mathbb{R}_{+}$, we have

$$
\begin{aligned}
& \mid\left((\psi(t)-\psi(0))^{1-\varsigma}\left(K u_{n}\right)(t)-\left((\psi(t)-\psi(0))^{1-\varsigma}(K u)(t) \mid\right.\right. \\
& \leq \mid v\left(t, u_{n}(t)\right)\left\{\frac{u_{0}}{v(0, u(0))}+\frac{(\psi(t)-\psi(0))^{1-\varsigma}}{\Gamma(\lambda)} \int_{0}^{t} \psi^{\prime}(s)(\psi(t)-\psi(s))^{\lambda-1} w\left(s, u_{n}(s)\right) d s\right\} \\
& -v(t, u(t))\left\{\frac{u_{0}}{v(0, u(0))}+\frac{(\psi(t)-\psi(0))^{1-\varsigma}}{\Gamma(\lambda)} \int_{0}^{t} \psi^{\prime}(s)(\psi(t)-\psi(s))^{\lambda-1} w(s, u(s)) d s\right\} \mid \\
& \leq \mid v\left(t, u_{n}(t)\right)\left\{\frac{u_{0}}{v(0, u(0))}+\frac{(\psi(t)-\psi(0))^{1-\varsigma}}{\Gamma(\lambda)} \int_{0}^{t} \psi^{\prime}(s)(\psi(t)-\psi(s))^{\lambda-1} w\left(s, u_{n}(s)\right) d s\right\} \\
& -v(t, u(t))\left\{\frac{u_{0}}{v(0, u(0))}+\frac{(\psi(t)-\psi(0))^{1-\varsigma}}{\Gamma(\lambda)} \int_{0}^{t} \psi^{\prime}(s)(\psi(t)-\psi(s))^{\lambda-1} w\left(s, u_{n}(s)\right) d s\right\} \\
& +v(t, u(t))\left\{\frac{u_{0}}{v(0, u(0))}+\frac{(\psi(t)-\psi(0))^{1-\varsigma}}{\Gamma(\lambda)} \int_{0}^{t} \psi^{\prime}(s)(\psi(t)-\psi(s))^{\lambda-1} w\left(s, u_{n}(s)\right) d s\right\} \\
& -v(t, u(t))\left\{\frac{u_{0}}{v(0, u(0))}+\frac{(\psi(t)-\psi(0))^{1-\varsigma}}{\Gamma(\lambda)} \int_{0}^{t} \psi^{\prime}(s)(\psi(t)-\psi(s))^{\lambda-1} w(s, u(s)) d s\right\} \mid
\end{aligned}
$$




$$
\begin{aligned}
& \leq\left|v\left(t, u_{n}(t)\right)-v(t, u(t))\right| \mid \frac{u_{0}}{v(0, u(0))}+\frac{(\psi(t)-\psi(0))^{1-\varsigma}}{\Gamma(\lambda)} \int_{0}^{t} \psi^{\prime}(s)(\psi(t)-\psi(s))^{\lambda-1} \\
& \times w\left(s, u_{n}(s)\right) d s|+| v(t, u(t)) \mid \frac{(\psi(t)-\psi(0))^{1-\varsigma}}{\Gamma(\lambda)} \\
& \times \int_{0}^{t} \psi^{\prime}(s)(\psi(t)-\psi(s))^{\lambda-1}\left|w\left(s, u_{n}(s)\right)-w(s, u(s))\right| d s .
\end{aligned}
$$

Hence

$$
\begin{aligned}
& \left|(\psi(t)-\psi(0))^{1-\varsigma}\left(K u_{n}\right)(t)-(\psi(t)-\psi(0))^{1-\varsigma}(K u)(t)\right| \\
& \leq\left|v\left(t, u_{n}(t)\right)-v(t, u(t))\right|\left\{\left|\frac{u_{0}}{v(0, u(0))}\right|\right. \\
& \left.+\frac{(\psi(t)-\psi(0))^{1-\varsigma}}{\Gamma(\lambda)} \int_{0}^{t} \psi^{\prime}(s)(\psi(t)-\psi(s))^{\lambda-1}\left|w\left(s, u_{n}(s)\right)\right| d s\right\} \\
& +L \frac{(\psi(t)-\psi(0))^{1-\varsigma}}{\Gamma(\lambda)} \int_{0}^{t} \psi^{\prime}(s)(\psi(t)-\psi(s))^{\lambda-1}\left|w\left(s, u_{n}(s)\right)-w(s, u(s))\right| d s .
\end{aligned}
$$

Case 1. If $t \in[0, d]$, then, in view of the facts that $u_{n} \rightarrow u$ as $n \rightarrow \infty, v$ and $w$ are continuous, by the Lebesgue dominated convergence theorem, from the equation (3.2), we have

$$
\left\|K\left(u_{n}\right)-K(u)\right\|_{B C_{\varsigma}} \rightarrow 0 \quad \text { as } \quad n \rightarrow \infty
$$

Case 2. If $t \in(d, \infty)$, then, from the hypotheses and (3.2), we have

$$
\begin{aligned}
& \left|(\psi(t)-\psi(0))^{1-\varsigma}\left(K u_{n}\right)(t)-(\psi(t)-\psi(0))^{1-\varsigma}(K u)(t)\right| \\
& \leq\left|v\left(t, u_{n}(t)\right)-v(t, u(t))\right|\left\{\left|\frac{u_{0}}{v(0, u(0))}\right|\right. \\
& \left.+\frac{(\psi(t)-\psi(0))^{1-\varsigma}}{\Gamma(\lambda)} \int_{0}^{t} \psi^{\prime}(s)(\psi(t)-\psi(s))^{\lambda-1} T(s) d s\right\} \\
& +2 L \frac{(\psi(t)-\psi(0))^{1-\varsigma}}{\Gamma(\lambda)} \int_{0}^{t} \psi^{\prime}(s)(\psi(t)-\psi(s))^{\lambda-1} T(s) d s .
\end{aligned}
$$

Then

$$
\begin{aligned}
& \left|(\psi(t)-\psi(0))^{1-\varsigma}\left(K u_{n}\right)(t)-(\psi(t)-\psi(0))^{1-\varsigma}(K u)(t)\right| \\
& \leq\left|v\left(t, u_{n}(t)\right)-v(t, u(t))\right|\left\{\left|\frac{u_{0}}{v(0, u(0))}\right|+\left((\psi(t)-\psi(0))^{1-\varsigma}\left(I_{0^{+}}^{\lambda ; \psi} T\right)(t)\right\}\right. \\
& +2 L\left((\psi(t)-\psi(0))^{1-\varsigma}\left(I_{0^{+}}^{\lambda ; \psi} T\right)(t) .\right.
\end{aligned}
$$

Since $u_{n} \rightarrow u$ as $n \rightarrow \infty, v$ is continuous and $(\psi(t)-\psi(0))^{1-\varsigma}\left(I_{0^{+}}^{\lambda ; \psi} T\right)(t) \rightarrow 0$ as $t \rightarrow \infty$, it follows from (3.3) that

$$
\left\|K\left(u_{n}\right)-K(u)\right\|_{B C_{\varsigma}} \rightarrow 0 \quad \text { as } \quad n \rightarrow \infty
$$

Step 2. $L\left(B_{R_{*}}\right)$ is uniformly bounded, and equicontinuous on every compact subset $[0, d]$ of $\mathbb{R}_{+}, d>0$. 
We have $L\left(B_{R_{*}}\right) \subset B_{R_{*}}$ and $B_{R_{*}}$ is bounded, so $L\left(B_{R_{*}}\right)$ is uniformly bounded.

Next, for each $t_{1}, t_{2} \in[0, d], t_{1}<t_{2}$, and $u \in B_{R_{*}}$, we have

$$
\begin{aligned}
& \left|\left(\psi\left(t_{2}\right)-\psi(0)\right)^{1-\varsigma}(K u)\left(t_{2}\right)-\left(\psi\left(t_{1}\right)-\psi(0)\right)^{1-\varsigma}(K u)\left(t_{1}\right)\right| \\
& \leq \mid v\left(t_{2}, u\left(t_{2}\right)\right)\left\{\frac{u_{0}}{v(0, u(0))}+\frac{\left(\psi\left(t_{2}\right)-\psi(0)\right)^{1-\varsigma}}{\Gamma(\lambda)} \int_{0}^{t_{2}} \psi^{\prime}(s)\left(\psi\left(t_{2}\right)-\psi(s)\right)^{\lambda-1} w(s, u(s)) d s\right\} \\
& -v\left(t_{1}, u\left(t_{1}\right)\right)\left\{\frac{u_{0}}{v(0, u(0))}+\frac{\left(\psi\left(t_{1}\right)-\psi(0)\right)^{1-\varsigma}}{\Gamma(\lambda)} \int_{0}^{t_{1}} \psi^{\prime}(s)\left(\psi\left(t_{1}\right)-\psi(s)\right)^{\lambda-1} w(s, u(s)) d s\right\} \mid \\
& \leq \mid v\left(t_{2}, u\left(t_{2}\right)\right)\left\{\frac{u_{0}}{v(0, u(0))}+\frac{\left(\psi\left(t_{2}\right)-\psi(0)\right)^{1-\varsigma}}{\Gamma(\lambda)} \int_{0}^{t_{2}} \psi^{\prime}(s)\left(\psi\left(t_{2}\right)-\psi(s)\right)^{\lambda-1} w(s, u(s)) d s\right\} \\
& -v\left(t_{1}, u\left(t_{1}\right)\right)\left\{\frac{u_{0}}{v(0, u(0))}+\frac{\left(\psi\left(t_{2}\right)-\psi(0)\right)^{1-\varsigma}}{\Gamma(\lambda)} \int_{0}^{t_{2}} \psi^{\prime}(s)\left(\psi\left(t_{2}\right)-\psi(s)\right)^{\lambda-1} w(s, u(s)) d s\right\} \\
& +v\left(t_{1}, u\left(t_{1}\right)\right)\left\{\frac{u_{0}}{v(0, u(0))}+\frac{\left(\psi\left(t_{2}\right)-\psi(0)\right)^{1-\varsigma}}{\Gamma(\lambda)} \int_{0}^{t_{2}} \psi^{\prime}(s)\left(\psi\left(t_{2}\right)-\psi(s)\right)^{\lambda-1} w(s, u(s)) d s\right\} \\
& -v\left(t_{1}, u\left(t_{1}\right)\right)\left\{\frac{u_{0}}{v(0, u(0))}+\frac{\left(\psi\left(t_{1}\right)-\psi(0)\right)^{1-\varsigma}}{\Gamma(\lambda)} \int_{0}^{t_{1}} \psi^{\prime}(s)\left(\psi\left(t_{1}\right)-\psi(s)\right)^{\lambda-1} w(s, u(s)) d s\right\} \mid .
\end{aligned}
$$

Thus

$$
\begin{aligned}
& \left|\left(\psi\left(t_{2}\right)-\psi(0)\right)^{1-\varsigma}(K u)\left(t_{2}\right)-\left(\psi\left(t_{1}\right)-\psi(0)\right)^{1-\varsigma}(K u)\left(t_{1}\right)\right| \\
& \leq\left|v\left(t_{2}, u\left(t_{2}\right)\right)-v\left(t_{1}, u\left(t_{1}\right)\right)\right| \frac{u_{0}}{v(0, u(0))} \\
& +\frac{\left(\psi\left(t_{2}\right)-\psi(0)\right)^{1-\varsigma}}{\Gamma(\lambda)} \int_{0}^{t_{2}} \psi^{\prime}(s)\left(\psi\left(t_{2}\right)-\psi(s)\right)^{\lambda-1} w(s, u(s)) d s \mid \\
& +\left|v\left(t_{1}, u\left(t_{1}\right)\right)\right| \frac{\left(\psi\left(t_{2}\right)-\psi(0)\right)^{1-\varsigma}}{\Gamma(\lambda)} \int_{0}^{t_{1}} \psi^{\prime}(s)\left(\psi\left(t_{2}\right)-\psi(s)\right)^{\lambda-1} w(s, u(s)) d s \\
& +\frac{\left(\psi\left(t_{2}\right)-\psi(0)\right)^{1-\varsigma}}{\Gamma(\lambda)} \int_{t_{1}}^{t_{2}} \psi^{\prime}(s)\left(\psi\left(t_{2}\right)-\psi(s)\right)^{\lambda-1} w(s, u(s)) d s \\
& -\frac{\left(\psi\left(t_{1}\right)-\psi(0)\right)^{1-\varsigma}}{\Gamma(\lambda)} \int_{0}^{t_{1}} \psi^{\prime}(s)\left(\psi\left(t_{1}\right)-\psi(s)\right)^{\lambda-1} w(s, u(s)) d s \mid .
\end{aligned}
$$

Hence

$$
\begin{aligned}
& \left|\left(\psi\left(t_{2}\right)-\psi(0)\right)^{1-\varsigma}(K u)\left(t_{2}\right)-\left(\psi\left(t_{1}\right)-\psi(0)\right)^{1-\varsigma}(K u)\left(t_{1}\right)\right| \\
& \leq\left|v\left(t_{2}, u\left(t_{2}\right)\right)-v\left(t_{1}, u\left(t_{1}\right)\right)\right|\left(\left|\frac{u_{0}}{v(0, u(0))}\right|\right. \\
& \left.+\frac{\left(\psi\left(t_{2}\right)-\psi(0)\right)^{1-\varsigma}}{\Gamma(\lambda)} \int_{0}^{t_{2}} \psi^{\prime}(s)\left(\psi\left(t_{2}\right)-\psi(s)\right)^{\lambda-1}|w(s, u(s))| d s\right) \\
& +L\left(\int_{0}^{t_{1}} \mid \frac{\left(\psi\left(t_{2}\right)-\psi(0)\right)^{1-\varsigma}}{\Gamma(\lambda)} \psi^{\prime}(s)\left(\psi\left(t_{2}\right)-\psi(s)\right)^{\lambda-1}\right. \\
& -\frac{\left(\psi\left(t_{1}\right)-\psi(0)\right)^{1-\varsigma}}{\Gamma(\lambda)} \psi^{\prime}(s)\left(\psi\left(t_{1}\right)-\psi(s)\right)^{\lambda-1} \mid \\
& \left.|w(s, u(s))| d s+\frac{\left(\psi\left(t_{2}\right)-\psi(0)\right)^{1-\varsigma}}{\Gamma(\lambda)} \int_{t_{1}}^{t_{2}} \psi^{\prime}(s)\left(\psi\left(t_{2}\right)-\psi(s)\right)^{\lambda-1}|w(s, u(s))| d s\right)
\end{aligned}
$$




$$
\begin{aligned}
& \leq\left|v\left(t_{2}, u\left(t_{2}\right)\right)-v\left(t_{1}, u\left(t_{1}\right)\right)\right|\left(\left|\frac{u_{0}}{v(0, u(0))}\right|\right. \\
& \left.+\frac{\left(\psi\left(t_{2}\right)-\psi(0)\right)^{1-\varsigma}}{\Gamma(\lambda)} \int_{0}^{t_{2}} \psi^{\prime}(s)\left(\psi\left(t_{2}\right)-\psi(s)\right)^{\lambda-1} T(s) d s\right) \\
& +L\left(\int_{0}^{t_{1}} \mid \frac{\left(\psi\left(t_{2}\right)-\psi(0)\right)^{1-\varsigma}}{\Gamma(\lambda)} \psi^{\prime}(s)\left(\psi\left(t_{2}\right)-\psi(s)\right)^{\lambda-1}\right. \\
& -\frac{\left(\psi\left(t_{1}\right)-\psi(0)\right)^{1-\varsigma}}{\Gamma(\lambda)} \psi^{\prime}(s)\left(\psi\left(t_{1}\right)-\psi(s)\right)^{\lambda-1} \mid \\
& \left.T(s) d s+\frac{\left(\psi\left(t_{2}\right)-\psi(0)\right)^{1-\varsigma}}{\Gamma(\lambda)} \int_{t_{1}}^{t_{2}} \psi^{\prime}(s)\left(\psi\left(t_{2}\right)-\psi(s)\right)^{\lambda-1} T(s) d s\right)
\end{aligned}
$$

From the continuity of the functions $T$ and $v$, by setting $T_{*}=\sup _{t \in[0, d]} T(t)$, we obtain

$$
\begin{aligned}
& \left|\left(\psi\left(t_{2}\right)-\psi(0)\right)^{1-\varsigma}(K u)\left(t_{2}\right)-\left(\psi\left(t_{1}\right)-\psi(0)\right)^{1-\varsigma}(K u)\left(t_{1}\right)\right| \\
& \leq\left|v\left(t_{2}, u\left(t_{2}\right)\right)-v\left(t_{1}, u\left(t_{1}\right)\right)\right|\left(\left|\frac{u_{0}}{v(0, u(0))}\right|+\frac{T_{*}\left(\psi\left(t_{2}\right)-\psi(0)\right)^{1-\varsigma}}{\Gamma(\lambda)} \int_{0}^{t_{2}} \psi^{\prime}(s)\left(\psi\left(t_{2}\right)-\psi(s)\right)^{\lambda-1} d s\right) \\
& +L T_{*}\left(\int_{0}^{t_{1}} \mid \frac{\left(\psi\left(t_{2}\right)-\psi(0)\right)^{1-\varsigma}}{\Gamma(\lambda)} \psi^{\prime}(s)\left(\psi\left(t_{2}\right)-\psi(s)\right)^{\lambda-1}\right. \\
& -\frac{\left(\psi\left(t_{1}\right)-\psi(0)\right)^{1-\varsigma}}{\Gamma(\lambda)} \psi^{\prime}(s)\left(\psi\left(t_{1}\right)-\psi(s)\right)^{\lambda-1} \mid d s \\
& \left.+\frac{\left(\psi\left(t_{2}\right)-\psi(0)\right)^{1-\varsigma}}{\Gamma(\lambda)} \int_{t_{1}}^{t_{2}} \psi^{\prime}(s)\left(\psi\left(t_{2}\right)-\psi(s)\right)^{\lambda-1} d s\right) \\
& \leq\left|v\left(t_{2}, u\left(t_{2}\right)\right)-v\left(t_{1}, u\left(t_{1}\right)\right)\right|\left(\left|\frac{u_{0}}{v(0, u(0))}\right|+\frac{T_{*}\left(\psi\left(t_{2}\right)-\psi(0)\right)^{1-\varsigma+\lambda}}{\Gamma(\lambda+1)}\right) \\
& +L T_{*}\left(\int_{0}^{t_{1}} \mid \frac{\left(\psi\left(t_{2}\right)-\psi(0)\right)^{1-\varsigma}}{\Gamma(\lambda)} \psi^{\prime}(s)\left(\psi\left(t_{2}\right)-\psi(s)\right)^{\lambda-1}\right. \\
& \left.-\frac{\left(\psi\left(t_{1}\right)-\psi(0)\right)^{1-\varsigma}}{\Gamma(\lambda)} \psi^{\prime}(s)\left(\psi\left(t_{1}\right)-\psi(s)\right)^{\lambda-1} \mid d s+\frac{\left(\psi\left(t_{2}\right)-\psi(0)\right)^{1-\varsigma}}{\Gamma(\lambda+1)}\left(\psi\left(t_{2}\right)-\psi\left(t_{1}\right)\right)^{\lambda}\right)
\end{aligned}
$$

As $t_{1} \rightarrow t_{2}$, the right-hand side of the inequality tends to zero.

Step 3. $L\left(B_{R}\right)$ is equiconvergent.

Let $u \in B_{R *}$. Then, for each $t \in \mathbb{R}_{+}$we have

$$
\begin{aligned}
& \left|(\psi(t)-\psi(0))^{1-\varsigma}(K u)(t)\right| \leq|v(t, u(t))|\left\{\left|\frac{u_{0}}{v(0, u(0))}\right|\right. \\
& \left.+\left|\frac{(\psi(t)-\psi(0))^{1-\varsigma}}{\Gamma(\lambda)} \int_{0}^{t} \psi^{\prime}(s)(\psi(t)-\psi(s))^{\lambda-1} w(s, u(s)) d s\right|\right\} \\
& \leq|v(t, u(t))|\left\{\left|\frac{u_{0}}{v(0, u(0))}\right|+\left|\frac{(\psi(t)-\psi(0))^{1-\varsigma}}{\Gamma(\lambda)} \int_{0}^{t} \psi^{\prime}(s)(\psi(t)-\psi(s))^{\lambda-1} T(s) d s\right|\right\} \\
& \leq L\left\{\left|\frac{u_{0}}{v(0, u(0))}\right|+(\psi(t)-\psi(0))^{1-\varsigma}\left(I_{0^{+}}^{\lambda ; \psi} T\right)(t)\right\} .
\end{aligned}
$$

Since

$$
(\psi(t)-\psi(0))^{1-\varsigma}\left(I_{0^{+}}^{\lambda ; \psi} T\right)(t) \rightarrow 0 \text { as } t \rightarrow+\infty
$$


we find

$$
|(K u)(t)| \leq L\left\{\left|\frac{u_{0}}{(\psi(t)-\psi(0))^{1-\varsigma} v(0, u(0))}\right|+\frac{(\psi(t)-\psi(0))^{1-\varsigma}\left(I_{0^{+}}^{\lambda ; \psi} T\right)(t)}{(\psi(t)-\psi(0))^{1-\varsigma}}\right\} .
$$

Hence,

$$
|(L u)(t)-(L u)(+\infty)| \rightarrow 0 \quad \text { as } \quad t \rightarrow+\infty,
$$

in view of Lemma 2.11 as a consequence of Steps $1-4$, we conclude that $K: B_{R_{*}} \rightarrow B_{R_{*}}$ is compact and continuous. Applying the Theorem 2.12, we have that $K$ has a fixed point $u$, which is a solution of problem (1.1) on $\mathbb{R}_{+}$.

Step 4. The uniform local attractivity of solutions.

We assume that $u_{*}$ is a solution of problem (1.1) under the conditions of this theorem. Set $u \in B\left(u_{*}, 2 L\left\{\left|\frac{u_{0}}{v(0, u(0))}\right|+2 T^{*}\right\}\right)$, we have

$$
\begin{aligned}
& \left|(\psi(t)-\psi(0))^{1-\varsigma}(K u)(t)-(\psi(t)-\psi(0))^{1-\varsigma}\left(u_{*}\right)(t)\right| \\
& \leq\left|(\psi(t)-\psi(0))^{1-\varsigma}(K u)(t)-(\psi(t)-\psi(0))^{1-\varsigma}\left(K u_{*}\right)(t)\right| \\
& \leq\left|v(t, u(t))-v\left(t, u_{*}(t)\right)\right|\left\{\left|\frac{u_{0}}{v(0, u(0))}\right|\right. \\
& \left.+\frac{(\psi(t)-\psi(0))^{1-\varsigma}}{\Gamma(\lambda)} \int_{0}^{t} \psi^{\prime}(s)(\psi(t)-\psi(s))^{\lambda-1}|w(s, u(s))| d s\right\} \\
& +L \frac{(\psi(t)-\psi(0))^{1-\varsigma}}{\Gamma(\lambda)} \int_{0}^{t} \psi^{\prime}(s)(\psi(t)-\psi(s))^{\lambda-1}\left|w(s, u(s))-w\left(s, u_{*}(s)\right)\right| d s \\
& \leq 2 L\left\{\left|\frac{u_{0}}{v(0, u(0))}\right|+\frac{(\psi(t)-\psi(0))^{1-\varsigma}}{\Gamma(\lambda)} \int_{0}^{t} \psi^{\prime}(s)(\psi(t)-\psi(s))^{\lambda-1} T(s) d s\right\} \\
& +2 L \frac{(\psi(t)-\psi(0))^{1-\varsigma}}{\Gamma(\lambda)} \int_{0}^{t} \psi^{\prime}(s)(\psi(t)-\psi(s))^{\lambda-1} T(s) d s \\
& \leq 2 L\left\{\left|\frac{u_{0}}{v(0, u(0))}\right|+2 T^{*}\right\} .
\end{aligned}
$$

Thus, we get

$$
\left\|K(u)-u_{*}\right\|_{B C_{\varsigma}} \leq 2 L\left\{\left|\frac{u_{0}}{v(0, u(0))}\right|+2 T^{*}\right\}
$$

So, we conclude that $K$ is a continuous function such that

$$
K\left(B\left(u_{*}, 2 L\left\{\left|\frac{u_{0}}{v(0, u(0))}\right|+2 T^{*}\right\}\right)\right) \subset B\left(u_{*}, 2 L\left\{\left|\frac{u_{0}}{v(0, u(0))}\right|+2 T^{*}\right\}\right) .
$$


Moreover, if $u$ is a solution of problem (1.1), then

$$
\begin{aligned}
\left|u(t)-u_{*}(t)\right| & =\left|(K u)(t)-\left(K u_{*}\right)(t)\right| \\
& \leq\left|v(t, u(t))-v\left(t, u_{*}(t)\right)\right|\left\{(\psi(t)-\psi(0))^{\varsigma-1}\left|\frac{u_{0}}{v(0, u(0))}\right|\right. \\
& \left.+\frac{1}{\Gamma(\lambda)} \int_{0}^{t} \psi^{\prime}(s)(\psi(t)-\psi(s))^{\lambda-1}|w(s, u(s))| d s\right\} \\
& +\frac{L}{\Gamma(\lambda)} \int_{0}^{t} \psi^{\prime}(s)(\psi(t)-\psi(s))^{\lambda-1}\left|w(s, u(s))-w\left(s, u_{*}(s)\right)\right| d s \\
& \leq 2 L\left\{(\psi(t)-\psi(0))^{\varsigma-1}\left|\frac{u_{0}}{v(0, u(0))}\right|\right. \\
& \left.+\frac{1}{\Gamma(\lambda)} \int_{0}^{t} \psi^{\prime}(s)(\psi(t)-\psi(s))^{\lambda-1}|w(s, u(s))| d s\right\} \\
& +\frac{L}{\Gamma(\lambda)} \int_{0}^{t} \psi^{\prime}(s)(\psi(t)-\psi(s))^{\lambda-1}\left|w(s, u(s))-w\left(s, u_{*}(s)\right)\right| d s \\
& \leq 2 L\left\{(\psi(t)-\psi(0))^{\varsigma-1}\left|\frac{u_{0}}{v(0, u(0))}\right|+2\left(I_{0^{+}}^{\lambda ; \psi} T\right)(t)\right\} .
\end{aligned}
$$

Therefore,

$$
\left|u(t)-u_{*}(t)\right| \leq 2 L\left\{(\psi(t)-\psi(0))^{\varsigma-1}\left|\frac{u_{0}}{v(0, u(0))}\right|+2 \frac{(\psi(t)-\psi(0))^{1-\varsigma}\left(I_{0^{+}}^{\lambda ; \psi} T\right)(t)}{(\psi(t)-\psi(0))^{1-\varsigma}}\right\} .
$$

By using (3.4) and the fact that

$$
\lim _{t \rightarrow \infty}(\psi(t)-\psi(0))^{1-\varsigma}\left(I_{0^{+}}^{\lambda ; \psi} T\right)(t)=0
$$

we conclude

$$
\lim _{t \rightarrow \infty}\left|u(t)-u_{*}(t)\right|=0 .
$$

Consequently, all solutions of problem (1.1) are uniformly locally attractive.

\section{An Example}

As an application of our results, we consider the following problem for a $\psi$-Hilfer fractional differential equation

$$
\left\{\begin{array}{l}
D_{0^{+}}^{\frac{1}{2}, \frac{1}{2} ; \psi} \frac{u(t)}{v(t, u(t))}=w(t, u(t)), \text { a.e. } \quad t \in \mathbb{R}_{+}, \\
\left.(\psi(t)-\psi(0))^{\frac{1}{4}} u(t)\right|_{t=0}=1,
\end{array}\right.
$$

where $\psi:[0,1] \rightarrow \mathbb{R}$ with $\psi(t)=\sqrt{t+3}$,

$$
\begin{gathered}
v(t, u)=\frac{1}{(1+t)(1+|u|)}, \\
\left\{\begin{array}{l}
w(t, u)=\frac{\beta(\psi(t)-\psi(0))^{\frac{-1}{4}} \sin t}{64(1+\sqrt{t})(1+|u|)}, t \in(0, \infty), \quad u \in \mathbb{R}, \\
w(0, u)=0, u \in \mathbb{R}
\end{array}\right.
\end{gathered}
$$


and

$$
\beta=\frac{9 \sqrt{\pi}}{16} .
$$

Clearly, the function $w$ is continuous. The hypothesis $\left(H_{2}\right)$ is satisfied with

$$
\left\{\begin{array}{l}
T(t)=\frac{\beta(\psi(t)-\psi(0))^{\frac{-1}{4}}|\sin t|}{64(1+\sqrt{t})}, \quad t \in(0, \infty), \\
T(0)=0 .
\end{array}\right.
$$

In addition, we have

$$
\begin{aligned}
(\psi(t)-\psi(0))^{\frac{1}{4}}\left(I_{0^{+}}^{\frac{1}{2} ; \psi} T\right)(t) & =\frac{(\psi(t)-\psi(0))^{\frac{1}{4}}}{\Gamma\left(\frac{1}{2}\right)} \int_{0}^{t} \psi^{\prime}(\tau)(\psi(t)-\psi(\tau))^{\frac{-1}{2}} T(\tau) d \tau \\
& \leq \frac{1}{4}(\psi(t)-\psi(0))^{\frac{-1}{4}} \rightarrow 0 \quad \text { as } \quad t \rightarrow \infty
\end{aligned}
$$

Simple computations show that all conditions of Theorem 3.2 are satisfied. Consequently, our problem (4.1) has at least one solution defined on $\mathbb{R}_{+}$, and all solutions of this problem are uniformly locally attractive.

\section{Conclusion}

In this paper, we provided some sufficient conditions ensuring the existence and the uniform locally attractivity of solutions of some $\psi$-Hilfer fractional differential equations. The technique used is based on Schauder's fixed point theory theorem.

\section{References}

[1] S. Abbas, and M. Benchohra, Advanced Functional Evolution Equations and Inclusions, Dev. Math., vol. 39, Springer, Cham, 2015.

[2] S. Abbas, and M. Benchohra, "Existence and stability of nonlinear fractional order RiemannLiouville, Volterra-Stieltjes multi-delay integral equations", J. Integ. Equat. Appl., vol. 25, pp. 143-158, 2013.

[3] S. Abbas, M. Benchohra, and T. Diagana, "Existence and attractivity results for some fractional order partial integrodifferential equations with delay", Afr. Diaspora J. Math., vol. 15, pp 87-100, 2013.

[4] S. Abbas, M. Benchohra, and J. Henderson, "Existence and attractivity results for Hilfer fractional differential equations", J. Math. Sci., vol. 243, 347-357, 2019. 
[5] S. Abbas, M. Benchohra, and G. M. N'Guérékata, Advanced Fractional Differential and Integral Equations, Nova Sci. Publ., New York, 2015.

[6] S. Abbas, M. Benchohra, and G. M.N' Guérékata, Topics in Fractional Differential Equations, Dev. Math., vol. 27, Springer, New York, 2015.

[7] S. Abbas, M. Benchohra, and J. J. Nieto, "Global attractivity of solutions for nonlinear fractional order Riemann-Liouville Volterra-Stieltjes partial integral equations", Electron. J. Qual. Theory Differ. Equat, vol. 81, pp. 1-15, 2012.

[8] R. Almeida, "Functional differential equations involving the [psi]-Caputo fractional derivative", Fractal and Fractional, vol. 4, no. 2, pp 1-8, 2020.

[9] B. Ahmad, S. K. Ntouyas, and J. Tariboon, "A nonlocal hybrid boundry value problem of Caputo fractional integro-differential equations", Acta Math. Sci. vol. 36, pp. 1631-1640, 2016.

[10] R. Almeida, "A Caputo, fractional derivative of a function with respect to another function", Comm. Nonlinear Sci. Numer. Simulat. vol. 44, pp. 460-481, 2017.

[11] C. Corduneanu, Integral Equations and Stability of Feedback Systems, Acad. Press, New York, 1973.

[12] B. C. Dhage, and V. Lakshmikantham, "Basic results on hybrid differential equations", Nonlinear Anal.: Hybrid Systems vol. 4, pp. 414-424, 2010.

[13] K. Diethelm, The analysis of fractional differential equations, Lecture Notes in Mathematics, Springer-verlag Berlin Heidelberg, 2010.

[14] S. Ferraoun, and Z. Dahmani, "Existence and stability of solutions of a class of hybrid fractional differential equations involving R-L-operator", J. Interd. Math., pp. 1-19, 2020.

[15] A. Granas, J. Dugundji, Fixed Point Theory, Springer-Verlag, New York, 2003.

[16] J. P. Kharade and K. D. Kucche, "On the impulsive implicit $\psi$-Hilfer fractional differential equations with delay", Math. Methods Appl. Sci., vol. 43, no 4, pp. 1938-1952, 2019.

[17] A. A. Kilbas, H. M. Srivastava, and J. J. Trujillo, Theory and Applications of Fractional Differential Equations, North-Holland Math Stud., 204, Elsevier, Amsterdam, 2006.

[18] K. D. Kucche, A. D. Mali, and J. V Sousa, "On the nonlinear $\Psi$-Hilfer fractional differential equations". Comput. Appl. Math., vol. 38, no. 2, paper no. 73, 25 pp, 2019.

[19] V. Lakshmikantham, and J. Vasundhara Devi, "Theory of fractional differential equations in a Banach space", Eur. J. Pure Appl. Math. vol. 1, pp. 38-45, 2008. 
[20] V. Lakshmikantham, and A. S. Vatsala, "Basic theory of fractional differential equations", Nonlin. Anal., vol. 69, pp. 2677-2682, 2008.

[21] V. Lakshmikantham, and A. S. Vatsala, "General uniqueness and monotone iterative technique for fractional differential equations", Appl. Math. Lett., vol. 21, pp. 828-834, 2008.

[22] K. Oldham, and J. Spanier, The Fractional Calculus, Academic Press, New York, 1974.

[23] I. Podlubny, Fractional Differential Equations, Mathematics in Science and Engineering, 198, Acad. Press, 1999.

[24] S. G. Samko, A. A. Kilbas, and O. I. Marichev, Fractional Integrals and Derivatives: Theory and Applications, Gordon Breach, Tokyo-Paris-Berlin, 1993.

[25] H. Sugumarana, R. W. Ibrahimb, and K. Kanagarajana, "On $\psi$-Hilfer fractional differential equation with complex order", Universal J. Math. Appl., vol. 1, no. 1, pp. 33-38, 2018.

[26] S. Sun, Y. Zhao, Z. Han, and Y. Li, "The existence of solutions for boundary value problem of fractional hybrid differential equations", Commun. Nonlinear Sci. Numer. Simulat., vol. 17, pp. 4961-4967, 2012.

[27] V. E. Tarasov, Fractional Dynamics: Application of Fractional Calculus to the Dynamics of Particles, Fields, and Media, Springer, Beijing-Heidelberg, 2010.

[28] J. Vanterler da C. Sousa, J. A. Tenreiro Machado, and E. Capelas de Oliveira, "The $\psi$-Hilfer fractional calculus of variable order and its applications", Comput. Appl. Math., vol. 39, no. 296 , pp. 1-38, 2020.

[29] J. Vanterler da C. Sousa, and E. Capelas de Oliveira, "On the $\psi$-Hilfer fractional derivative", Commun. Nonlinear Sci. Numer. Simulat., vol. 60, pp. 72-91, 2018.

[30] J. Vanterler da C. Sousa, and E. Capelas de Oliveira, "On the -fractional integral and applications", Comput. Appl. Math., vol. 38, no. 4, pp. 1-22, 2019.

[31] Y. Zhao, S. Sun, Z. Han, and Q. Li, "Theory of fractional hybrid differential equations", Comput. Math. Appl., vol. 62, pp. 1312-1324, 2011.

[32] Y. Zhou, Basic Theory of Fractional Differential Equations, World Scientific, Singapore, 2014.

[33] Y. Zhou, Fractional Evolution Equations and Inclusions: Analysis and Control, Elsevier, Acad. Press, 2016.

[34] Y. Zhou, J. Wang, and L. Zhang, Basic Theory of Fractional Differential Equations, Second Edition, World Scientific, Singapore, 2017. 